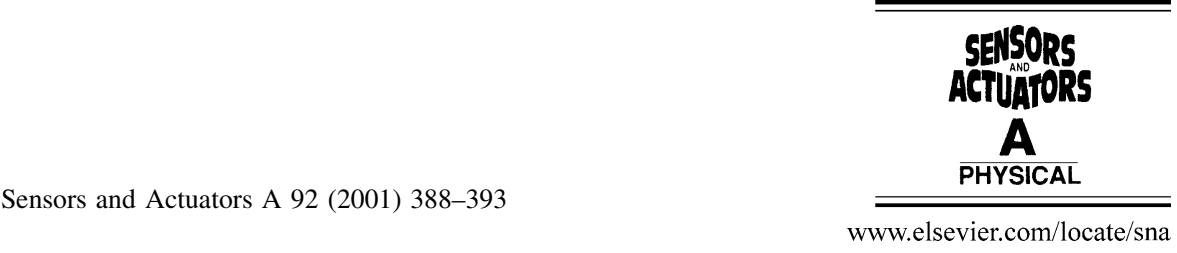

\title{
Powder blasting for the realisation of microchips for bio-analytic applications
}

\author{
D. Solignac ${ }^{\mathrm{a}, *}$, A. Sayah ${ }^{\mathrm{a}}$, S. Constantin ${ }^{\mathrm{b}}$, R. Freitag ${ }^{\mathrm{b}}$, M.A.M. Gijs ${ }^{\mathrm{a}}$ \\ ${ }^{\mathrm{a}}$ Institute of Microsystems, Swiss Federal Institute of Technology Lausanne (EPFL), CH-1015 Lausanne, Switzerland \\ ${ }^{\mathrm{b}}$ Center of Biotechnology, Swiss Federal Institute of Technology Lausanne (EPFL), CH-1015 Lausanne, Switzerland
}

\begin{abstract}
We introduce powder blasting for the fabrication of glass microchips. Powder blasting is a fast and cheap technique with which we pattern channels in sodalime and pyrex glass with a width down to $100 \mu \mathrm{m}$. We combine the technique with appropriate bonding procedures to realise sealed microchannel structures. We study the transport of fluorescent dye solutions and fluorescent beads within channels made by powder blasting and in 'classical' channels made by HF-etching. We find a remarkable difference in sign of the electric field induced flow for both types of channels and explain the observed strong plug broadening effects in the powder blasted channels. (C) 2001 Elsevier Science B.V. All rights reserved.
\end{abstract}

Keywords: Electrophoresis; Powder blasting; Glass microchip; Micromachining; Low temperature bonding; Fluorescent beads; Electro-osmotic flow.

\section{Introduction}

The integration of chemical analysis devices on-chip, especially electrophoresis devices, is a research area of increasing importance today [1]. Si substrates or polymer moulded structures have been used for the fabrication of capillary electrophoresis microchips [2], but mostly glass is used due to its low auto-fluorescence and good electrical isolation. Currently, the technologies employed for the micromachining of glass microfluidic systems are rather limited and mostly rely on aggressive chemical processes, like HF etching after a standard photolithographic process $([3,4]$ and literature cited therein). The objective of our work is to contribute with a fast and cheap technology for the realisation of glass microchips, which does not require a clean room environment.

We use powder blasting [5] as a very efficient and fast technique for the mechanical etching and drilling of glass. We combine this new microstructuring technique with a recently developed pressure-assisted low-temperature bonding process for sodalime glass slides [6] to realise sealed microchannels. We have compared the electro-osmotic flow (EOF) properties of powder blasted channels with those of 'classical' HF-etched channels by studying the electric field induced velocity of fluorescent beads within the channels.

\footnotetext{
${ }^{*}$ Corresponding author. Fax: +41-21-693-59-50.
}

We find beads going in opposite directions due to different electro-osmotic mobilities between powder blasted and HFetched channels. We also find a strong plug broadening effect in the powder blasted channels. This may render powder blasting less attractive for electrophoretic separation applications.

\section{Microfabrication}

Micropatterning by powder blasting relies on the mechanical erosion of a masked substrate by a high velocity powder beam originating from a pressurised nozzle. The size of the powder particles is around $30 \mu \mathrm{m}$. A contact mask with high wear resistance, like a metal, allows to micro-pattern structures with minimum sizes of about $50 \mu \mathrm{m}$. With more than $100 \mu \mathrm{m} / \mathrm{min}$, the etching rate of the powder blasting process is much higher than the conventional wet chemical etching of glass. Moreover, due to this high speed, powder blasting is an ideal technique for the fabrication of access holes, forming the interconnection of the microchannel to the external world.

Fig. 1 is a photograph of a microchip realised by patterning a cross-shaped microchannel in a sodalime glass microscope slide and by bonding it with a second slide. Access holes are drilled in the upper glass slide by powder blasting, after which fluidic reservoirs of poly-dimethylsiloxane (PDMS) with a volume of $60 \mathrm{~mm}^{3}$ are glued on the chip 


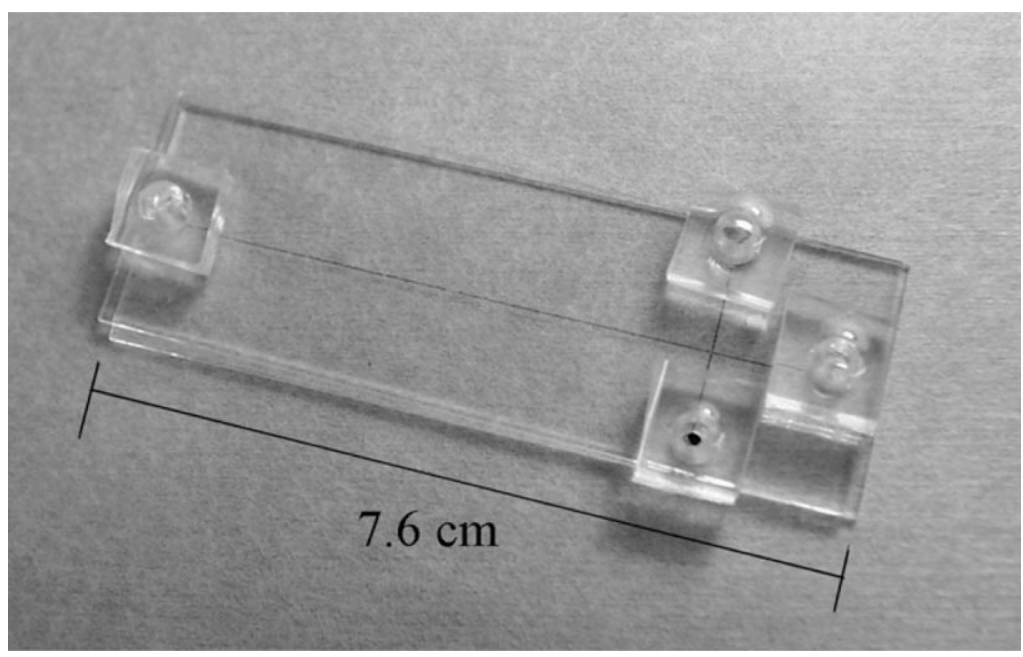

Fig. 1. Photograph of a capillary electrophoresis chip composed of two microstructured sodalime microscope slides bonded on top of each other. Moulded reservoirs in PDMS are glued on the four access holes.

using liquid PDMS. The shorter channel allows the introduction and the injection of a sample plug in the longer separation channel. The powder blasted channels are $100 \mu \mathrm{m}$ wide and $30 \mu \mathrm{m}$ deep. The roughness of the channel is measured using a profilometer and is in the $8-10 \mu \mathrm{m}$ range. A scanning electron microscope (SEM) photograph of the cross-section of the bonded structure is shown in Fig. 2a and an SEM photograph of a powder blasted access hole is shown in Fig. 2b. Fig. 3 shows the surface topography of the channel system on the sodalime glass slide, as measured with a micro-optical three-dimensional (3D)measuring system GFM [7]. For functional comparison, we also realised microchips with HF-etched microchannels after a standard photolithographic process. In this case, the roughness of the channel obtained is comprised between 30 and $60 \mathrm{~nm}$, according to atomic force microscopy measurements.

After the etching of the microchannels, the bonding with the cover slide is as follows: first of all, a cleaning step of the two slides is necessary; the cleaning process consists of washing with acetone, then with a Piranha solution (three volumes of sulphuric acid and one volume of hydrogen peroxide) during $30 \mathrm{~min}$. at room temperature. The resulting hydrophilised slides are rinsed with de-ionised water and brought into contact for subsequent bonding.

Besides the well known fusion bonding at temperatures of about $600^{\circ} \mathrm{C}$, we use a new pressure-assisted low temperature bonding process to bond the two sodalime microscope slides at low temperature (down to $100^{\circ} \mathrm{C}$ ). The pressure is applied by a Carver 4120 hot press, allowing to apply weights up to $11 \mathrm{t}$ at temperatures up to $340^{\circ} \mathrm{C}$. We study, in particular, the time and the pressure dependence of the bonding by evaluating bonding strengths with a pull test machine. The result is shown in Fig. 4. We note the very high bonding strengths (6 MPa) at unprecedented low temperatures. Moreover, by increasing the annealing temperature at $200^{\circ} \mathrm{C}$, the bonding strength reaches $10 \mathrm{MPa}$, which is higher or of the order of the best values obtain by HFassisted [8] or plasma-assisted bonding [9].

\section{Experimental results and discussion}

We study the EOF properties of the microchannels by applying a high field (up to $2.5 \mathrm{kV} / \mathrm{cm}$ ) over the separation channel. Microscopic observation of the flow of a fluorescein dye solution in powder blasted channels shows the dye flowing to the anode. On the other hand, when the same experiment is done using a HF etched microchannel, the dye flow is directed to the cathode. In a subsequent experiment, we study the field-induced flow of a fluorescein-isothiocyanate (FITC) labeled amino-acid mixture in $50 \mathrm{mM}$ borate buffer $(\mathrm{pH}=9.2)$. This solution is injected by pressure in the short channel and a sample plug is driven in the separation channel under the application of the electric field. Fig. 5 shows the evolution in time of the shape of this plug. After a few seconds, we observe a strong diffusion and broadening of the plug, which precludes any chance of electrophoretic separation. On the other hand, the same amino-acid mixture was easily separated into a commercial capillary electrophoresis system. Apparently, the surface state of the powder blasted microchannel is determining, to a large extent, the apparent mobility of the liquid.

To understand and to quantify the EOF properties in both powder blasted and HF-etched channels, we study the transport of fluorescent beads bearing negative charge (diameter $2 \mu \mathrm{m}$, Molecular Probes). Within the channel, the effective bead mobility is composed of an electrophoretic mobility due to the presence of a charge in an electrical field, and of an electro-osmotic mobility, arising from the double layer wall effect. To characterise the electrophoretic velocity of the fluorescent beads, we first performed electric fieldinduced flow experiments in a beaker glass, as schematically shown in Fig. 6a. Two planar Pt electrodes are plunged in a 


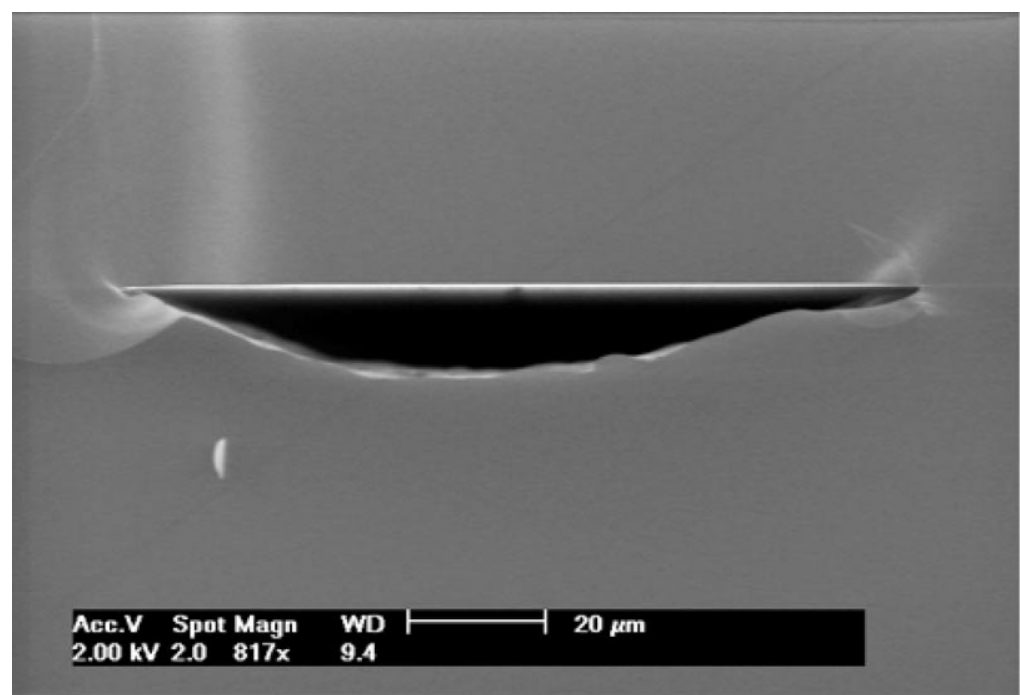

(a)

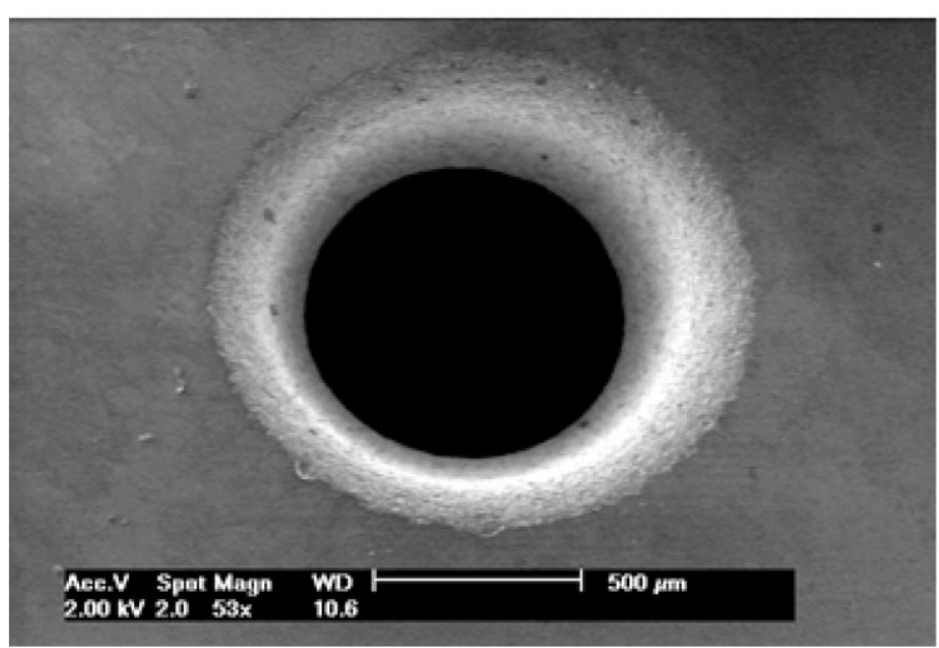

(b)

Fig. 2. (a) SEM photograph of a cross section of a $100 \mathrm{~mm}$ wide powder blasted channel after the bonding with a cover glass; (b) SEM photograph of a $1 \mathrm{~mm}$ diameter hole drilled in glass by powder blasting to provide a channel access port.

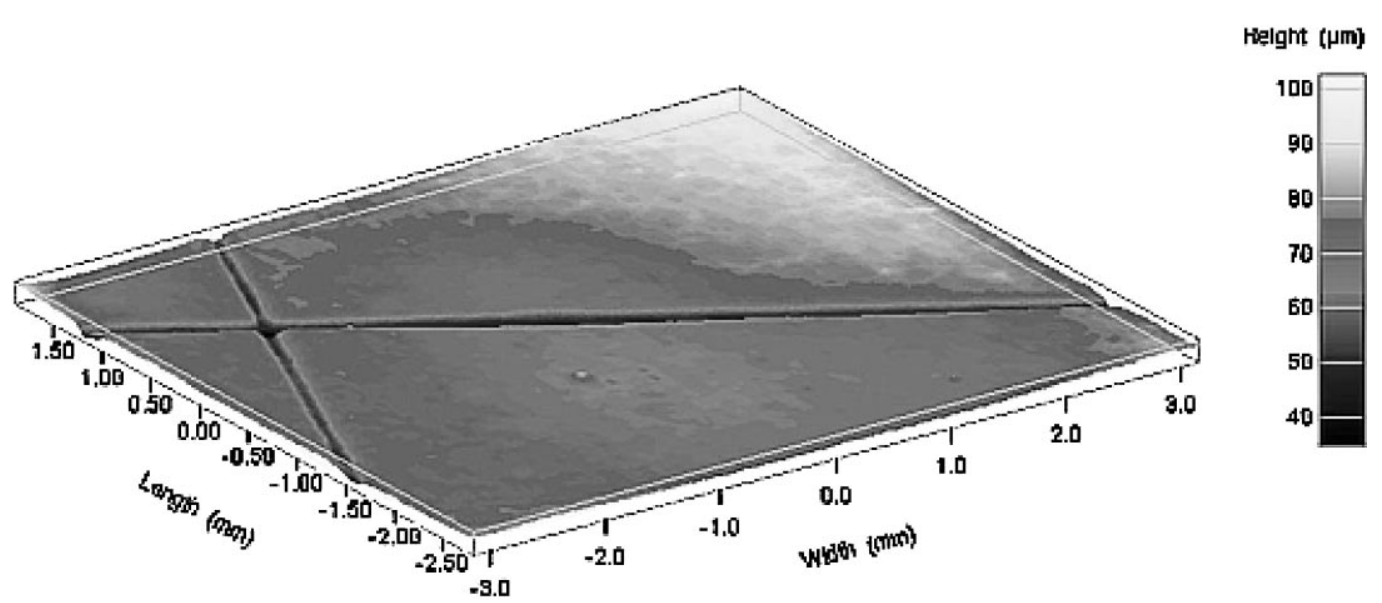

Fig. 3. Three-dimensional view of the surface topography of a sodalime glass slide with crossing powder blasted channels. 


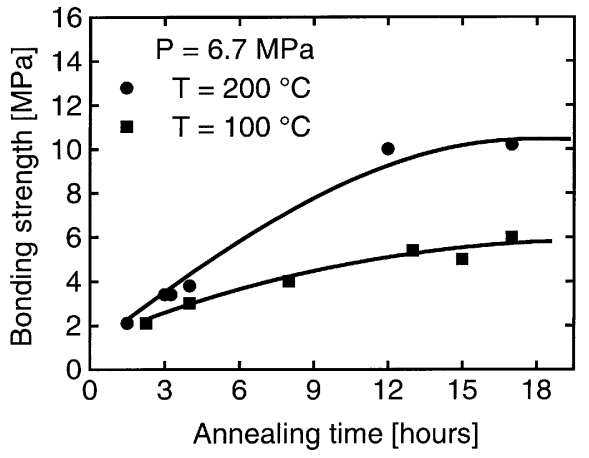

Fig. 4. Annealing time dependence on the bonding strength of two sodalime glass slides at $T=200^{\circ} \mathrm{C}$ and $T=100^{\circ} \mathrm{C}$ with a $6.7 \mathrm{MPa}$ applied pressure.

$10 \mathrm{mM}$ borate buffer solution $(\mathrm{pH}=9.2)$ and placed parallel to each other at a distance of $0.5 \mathrm{~cm}$. Evidently, in such geometry, there is no influence of any wall or double layer effects, as one is in the bulk of the solution. The velocity of the beads is measured at various voltages under ultra-violet illumination, whereby their fluorescent signal is observed by a CCD camera mounted under the microscope. Fig. $6 \mathrm{~b}$ shows a schematic diagram of the corresponding experiment in a microchannel. Here, due to the presence of the channel wall, both electrophoretic and electro-osmotic flow effects evidently play a role.

Fig. 7a shows the average bead velocity as a function of electric field for the beaker glass experiment of Fig. 6a. The negatively charged fluorescent beads, when placed in the electric field $\mathrm{E}$, start to move in the direction of the anode. We assume that the electrophoretic mobility $\left(\mu_{\mathrm{EP}}\right)$ of the beads is equivalent to the apparent mobility of the beads, as expressed by

$\mu_{\mathrm{EP}}=\frac{v_{\mathrm{B}}}{E}$

with $v_{\mathrm{B}}$ the measured velocity of the beads in the beaker and $E$ the electric field. We find that the electrophoretic mobility $\mu_{\mathrm{EP}}=7.0 \mathrm{~cm}^{2} \mathrm{kV}^{-1} \mathrm{~s}^{-1}$, in the $0-20 \mathrm{~V} / \mathrm{cm}$ range. Above $20 \mathrm{~V} / \mathrm{cm}$, the non-linear curve is attributed to Joule heating effects.

Having this reference value for the electrophoretic mobility of the beads, we have carried out electric field transport experiments under equivalent conditions in both powder blasted channels and HF etched channels with the same cross-section (around $1900 \mu \mathrm{m}^{2}$ ). The results are presented in Fig. 6b. A positive velocity corresponds to beads flowing in the direction of the positive electrode and a negative velocity to beads going to the opposite side. We obtain the remarkable result that, depending on the type of channel (powder blasted or HF-etched), the beads are transported in opposite directions under the same experimental conditions. We also note the absence of Joule heating up to large electrical fields $(2 \mathrm{kV} / \mathrm{cm})$.

The explanation for the different sign in bead velocity is as follows: in the microchannels etched in glass, the EOF (always directed toward the cathode) is opposed to the
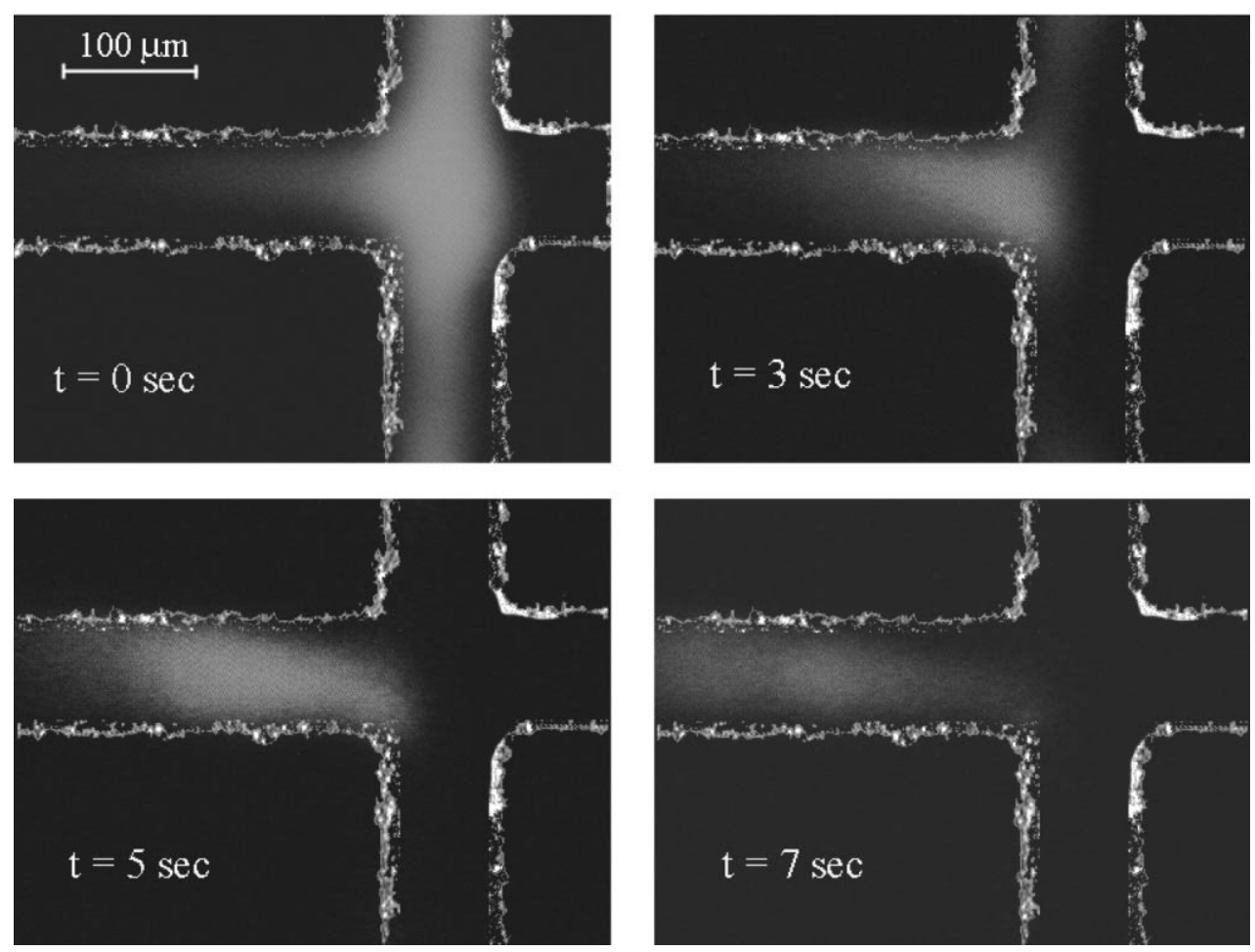

Fig. 5. Photograph of the time evolution of a plug when applying a voltage along the powder blasted separation channel. The plug is composed of a fluorescently labelled amino acid mixture in a $50 \mathrm{mM}$ borate buffer solution. 


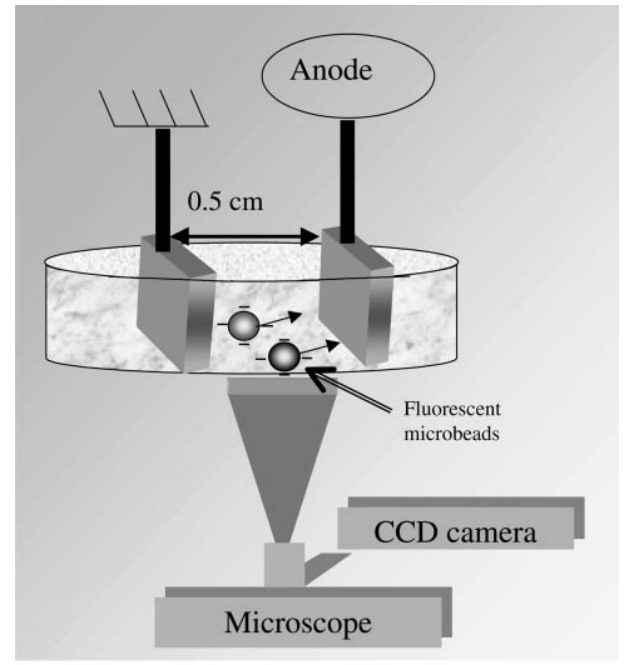

(a)

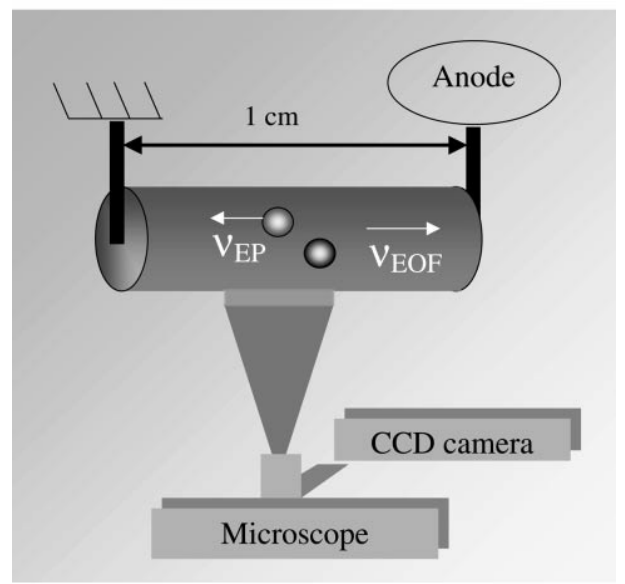

(b)

Fig. 6. (a) Schematic of the set-up used for the electrophoretic mobility measurement of the fluorescent microbeads in a beaker glass; (b) schematic view of the set-up used to study the transport of the fluorescent microbeads within the channels.

electrophoretic flow of the negatively charged beads. We can express the apparent or total mobility of the beads in the microchannel $\left(\mu_{\mathrm{Tot}}\right)$ as

$\mu_{\mathrm{Tot}}=\mu_{\mathrm{EP}}-\mu_{\mathrm{EOF}}=\frac{v_{\mathrm{C}}}{E}$

with $\mu_{\mathrm{EOF}}$ the electro-osmotic mobility in the channel and $v_{\mathrm{C}}$ the measured velocity of the beads in the channel. We should note that we have always used low bead concentrations $(<0.002 \mathrm{wt} . \%)$, preventing any surface charge redistribution or electrical polarisation effects of the beads in solution.

Using the value for the electrophoretic mobility of the beads, as measured in the beaker glass, we estimate the EOF mobility for electric fields below $2 \mathrm{kV} / \mathrm{cm}$, in both types of channels. For the HF-etched microchannels, $\mu_{\mathrm{EOF}}=$ $7.8 \mathrm{~cm}^{2} \mathrm{kV}^{-1} \mathrm{~s}^{-1}$. For powder blasted channels, one finds a value of $\mu_{\mathrm{EOF}}=3.4 \mathrm{~cm}^{2} \mathrm{kV}^{-1} \mathrm{~s}^{-1}$. We note a decrease of the electro-osmotic mobility in the powder blasted channels
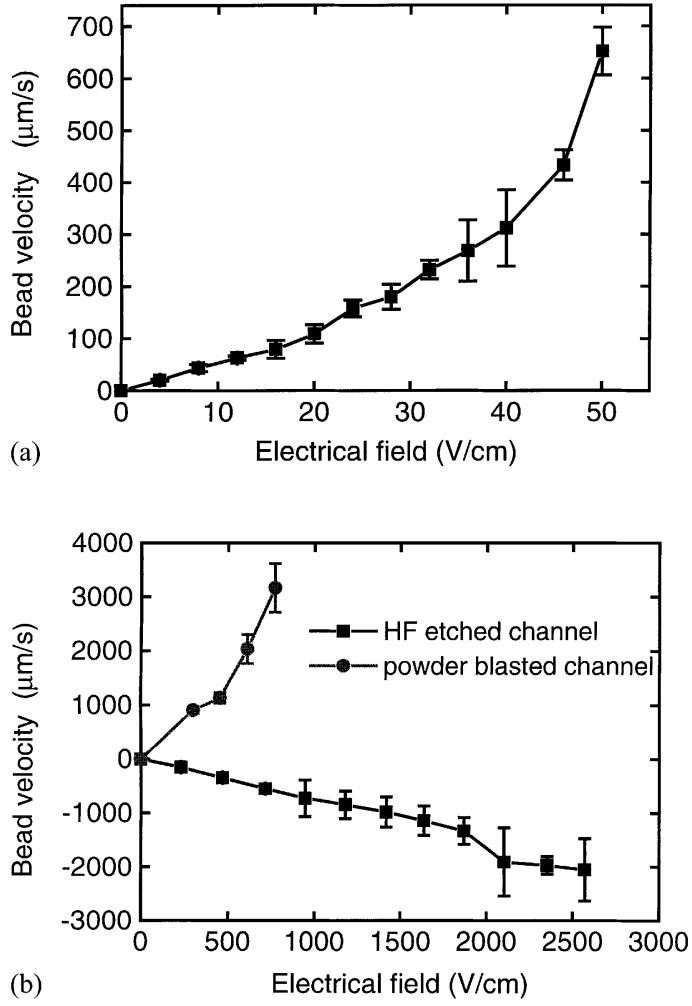

Fig. 7. (a) Measurement of the electrophoretic bead velocity as a function of the electric field in a beaker containing a $10 \mathrm{mM}$ borate buffer solution; (b) bead velocity measured in both powder blasted and HF-etched channels with $10 \mathrm{mM}$ borate buffer solution.

of more than $50 \%$ with respect to the value found for the HFetched channels. A probable explanation for this phenomenon is the difference in channel wall roughness obtained from the two microfabrication methods: EOF is clearly very sensitive to the physical state of the channel surface [10].

This result may also explain the difference in sign of the velocity of the fluorescein dye solution in powder blasted and HF-etched channels. Indeed, the fluorescein molecule has a certain charge/size ratio (similar to the microbeads), resulting in an electrophoretic mobility somewhat larger than the electro-osmotic mobility in the powder blasted channel. For the HF-etched channels, this is not the case. Also, the nearly opposite mobilities $\mu_{\mathrm{EOF}}$ and $\mu_{\mathrm{EP}}$ result in a very small flow velocity in the powder blasted channels, as found experimentally during the plug injection experiment of Fig. 5. Finally, we can say that the experiments with the microbeads allow us to understand the differences in transport behaviour of powder blasted and HF-etched channels.

\section{Conclusions}

We have combined powder blasting a very efficient and fast technique for the mechanical etching and drilling of glass with a recently developed pressure-assisted lowtemperature bonding process, to realise microchannels for 
fluidic bio-analytical applications. We have compared the electro-osmotic flow properties of powder blasted channels with those of HF-etched channels by studying the electric field induced transport of fluorescent dye solutions, FITClabeled amino acids, and fluorescent beads. We find opposing bead and dye solution velocities in powder blasted and HF-etched channels, due to different electro-osmotic mobilities in both systems. The nearly compensating electrophoretic and electro-osmotic flow mobilities in the powder blasted channels also explain the small total mobility and, in part, the strong observed plug broadening effect.

\section{References}

[1] D.J. Harrison, A. Van den Berg (Eds.), Micro Total Analysis Systems'98, Kluwer Academic Publishers, Dordrecht, 1998.

[2] S.C. Wang, M.D. Morris, Plastic microchip electrophoresis with analyte velocity. Application to fluorescence background rejection, Anal. Chem. 72 (2000) 1448.

[3] C.S. Effenhauser, A. Manz, H.M. Widmer, Glass chips for high-speed capillary electrophoresis separations with submicrometer plate heights, Anal. Chem. 65 (1993) 2637.
[4] S.C. Jacobson, A.W. Moore, J.M. Ramsey, Fused quartz substrates for microchip electrophoresis, Anal. Chem. 67 (1995) 2059.

[5] E. Belloy, S. Thurre, E. Walckiers, A. Sayah, M.A.M. Gijs, The introduction of powder blasting for sensor and microsystem applications, Sens. Actuators A 84 (2000) 330.

[6] A. Sayah, D. Solignac, T. Cueni, M.A.M. Gijs, Development of novel low-temperature bonding technologies for microchip chemical analysis applications, Sens. Actuators A 84 (2000) 103.

[7] Product information MikroCAD ${ }^{\circledR}$, GFMesstechnik GmbH, Berlin, Germany.

[8] H. Nakanishi, T. Nishimoto, M. Kanai, T. Saitoh, K. Nakamura, S. Shoji, Condition optimisation, reliability evaluation of $\mathrm{SiO}_{2}-\mathrm{SiO}_{2}$ $\mathrm{HF}$ bonding and its application for UV detection micro flow cell, in: Proceedings of the Transducers 99, Sendai, Japan, 1999, p.1332.

[9] H. Takagi, R. Maeda, N. Hosoda, T. Suga, Integration of dissimilar materials room-temperature wafer bonding using Ar beam surface activation, in: Proceedings of the Transducers 99, Sendai, Japan, 1999, p.1328.

[10] R. Schasfoort, R. Guijt-van Duijn, S. Schlautmann, H. Frank, H. Billiet, G. Van Dedem, A. Van Den Berg, Miniaturized capillary electrophoresis system with integrated conductivity detector, in: A. Van den Berg, W. Olthuis, P. Bergveld (Eds.), Micro Total Analysis Systems 2000, Kluwer Academic Plublishers, Dordrecht, 2000, p. 391 . 